\title{
Post-Operative Cluster Headache Following Carotid Endarterectomy
}

\author{
Thijs H.T. Dirkx Peter J. Koehler \\ Department of Neurology, Zuyderland Medical Centre, Heerlen, The Netherlands
}

\section{Keywords}

Cluster headache - Carotid endarterectomy .

Secondary cluster headache · Pathophysiology

of cluster headache

\begin{abstract}
Background: Secondary cluster headache following carotid endarterectomy (CEA) is a rare condition and may help us understand the pathophysiology of primary cluster headache. Summary: We describe 2 patients diagnosed with cluster headache, fulfilling the ICHD-IIIB criteria, following CEA. Neither of the patients had headache prior to surgery. They both responded to treatment with oxygen and verapamil. Recent medical literature does not describe any definite cases of cluster headache following CEA. Cluster-like headache has been reported in several studies in the 1990s. Recent studies in primary cluster headache patients show evidence for a central origin of cluster headache in which no peripheral drive seems necessary. Key Messages: Our findings may provide more insight into the pathophysiology and show how a peripheral cause may lead to cluster headache. We hypothesize a role of the trigemino-autonomic reflex. Damage to the carotid artery may activate this reflex and trigger cluster headache. Injury to the internal carotid artery may unleash attacks in patients who are predisposed to develop cluster headache. Further study on the subject is needed to resolve this issue.

(c) 2017 S. Karger AG, Basel
\end{abstract}

\section{Introduction}

Cluster headache is one of the primary headache types with a prevalence of approximately $0.1 \%$ [1].

Much of the pathophysiology still remains unclear. Recent studies show an important role for the posterior hypothalamus, which may be the primary trigger area for cluster headache [2].

The clinical symptoms may be explained by the activation of the trigemino-autonomic (TGA) reflex. Stimulation of the trigeminal nerve roots may lead to localized pain and by way of the TGA-reflex to secondary autonomic symptoms and vasodilatation. This reflex is also present in healthy persons. It is possible that a disturbance in the posterior hypothalamus may disinhibit this reflex leading to the clinical syndrome of cluster headache [2]. Cluster headache occurring secondary to various kinds of disorders has been described in case reports and a recent review [3].

We describe 2 patients who developed cluster headache following a carotid endarterectomy (CEA).

\section{Case Reports}

Patient A

A 67-year-old man presented with lacunar infarction of the left hemisphere. Carotid artery duplex showed a significant (over 70\%) bilateral stenosis of the internal carotid artery (ICA). Two weeks after presentation, CEA of the left ICA was performed. The proce-

\section{KARGER}

๑ 2017 S. Karger AG, Basel

E-Mail karger@karger.com

www.karger.com/ene
Thijs H.T. Dirkx

Department of Neurology

Zuyderland Medical Centre

PO Box 4446, NL-6401 CX Heerlen (The Netherlands)

E-Mail t.dirkx@zuyderland.nl 
Table 1. Summary of literature review

\begin{tabular}{|c|c|c|c|c|c|c|c|}
\hline Series & Number & $\begin{array}{l}\text { Mean age, } \\
\text { years }\end{array}$ & Male, $n(\%)$ & $\begin{array}{l}\text { Incidence of } \\
\text { headache, } n(\%)\end{array}$ & $\begin{array}{l}\text { Ipsilateral pain, } \\
n(\%)\end{array}$ & $\begin{array}{l}\text { "Cluster-like" } \\
\text { headache, } n(\%)\end{array}$ & $\begin{array}{l}\text { Cluster } \\
\text { headache, } n(\%)\end{array}$ \\
\hline Messert and Black [7], 1978 & 57 & ? & ? & $24(42)$ & $9(38)$ & $5(21)$ & $1(4)^{* *}$ \\
\hline Tehindrazanarivelo et al. [5], 1992 & 50 & 70 & $36(72)$ & $31(62)$ & $8(26)$ & $0(0)$ & $0(0)$ \\
\hline Albuquerque et al. [4], 2012 & 32 & 67.3 & $22(69)$ & $12(38)$ & $11(92)$ & $0(0)$ & $0(0)$ \\
\hline Total & 193 & $66.3^{*}$ & $98(72)^{*}$ & $88(46)$ & $44(50)$ & $21(24)$ & $1(1)^{*}$ \\
\hline
\end{tabular}

* Inadequate data in some studies. ${ }^{* *}$ Inadequate data given but one patient possibly fulfilling the ICHD-IIIB criteria.

dure was performed without complications. Two days after surgery, our patient developed bifrontal headache without hypertension and a hyperperfusion syndrome was excluded. A few days later, the headache changed and the typical characteristics of cluster headache became evident. Carotid artery duplex did not show restenosis or dissection. In the months following surgery, he had headache attacks, lasting 30-90 min, twice a day. The pain was localized around the left eye and accompanied by a red, tearing eye, rhinorrhea, restlessness and photophobia. Neurologic examination showed no abnormalities. An MRI scan of the brain showed old ischemic lesions and was otherwise unremarkable. Notably, no abnormalities were found in the hypothalamic region. The patient was diagnosed with cluster headache, fulfilling the ICHD-IIIB criteria. He responded to oxygen and his attack frequency diminished after starting verapamil. A few weeks later, he had no more attacks, but they reoccurred as soon as the verapamil dose was decreased. After 8 months, the attacks had completely ceased and verapamil could be stopped.

\section{Patient $B$}

A 63-year-old man was diagnosed with ischemic stroke of the left hemisphere. MRI showed recent infarction in the left frontal lobe. Carotid artery duplex showed a significant (over 70\%) stenosis of the left ICA, for which CEA was performed. Seven days postsurgery he developed left-sided headache, which occurred as nightly attacks of 15-30 min. There was a throbbing pain behind the left eye, accompanied by restlessness and left-sided nasal congestion. Neurologic examination was unchanged. An MRI scan of the brain showed no new ischemic lesions and a normal hypothalamic region. MRA showed no stenosis or dissection of the left ICA. He fulfilled the ICHD-IIIB criteria for cluster headache. $\mathrm{He}$ responded well to oxygen treatment and his attacks disappeared within 2 months after starting verapamil.

\section{Literature Review}

A literature search was conducted using PubMed. We searched for "(cluster) headache" and "carotid endarterectomy." We included English language articles only. No limits with regard to publication date were placed on the search. Also, we looked for additional articles in the refer- ence lists of identified articles. Recent medical literature does not describe post-operative cluster headache following CEA. Cluster-like headache, however, has been reported in several prospective studies and case reports in the 1990s, which we discuss briefly. A summary of headache following CEA is given in Table 1.

Albuquerque et al. [4] and Tehindrazanarivelo et al. [5] studied the occurrence of headache following CEA. They do not describe any patients who developed cluster headache or a cluster-like headache following CEA. They mostly describe a mild headache occurring after surgery.

In the 2012 study by Albuquerque et al. [4], 38\% of the 32 patients developed headache following CEA, usually ipsilaterally and in all cases described as mild. No severe ipsilateral pain or cluster headache was described.

Tehindrazanarivelo et al. [5] included 50 patients who underwent CEA. They were followed up to 1 month after surgery and $62 \%$ of the patients developed headache. Usually it was a mild headache for which no treatment was necessary. Neither autonomic symptoms nor Horner's syndrome was described. No severe ipsilateral headache, "cluster-like" headache or cluster headache was found either.

On the contrary, De Marinis et al. [6] described 21 out of 54 patients developing post-CEA headache, of whom 16 had cluster-like headache. A definition of this clusterlike headache was not provided. Four patients, however, were described, who developed autonomic symptoms and another 4 presenting with Horner's syndrome. There may have been patients fulfilling the ICHD-criteria for cluster headache; however, not enough data was provided. Post-operative pupillary testing in this study showed a decreased oculosympathetic activity, ipsilateral to the side of the headache, in all patients with headache, compared to $50 \%$ in those without headache. This suggests the involvement of the sympathetic plexus around the ICA. 
Table 2. ICHD-IIIB criteria [9]

Post-endarterectomy headache

Headache caused by the surgical procedure of carotid endarterectomy. Pain can also involve the neck and face. It can remain isolated or be a warning symptom preceding the focal deficits of (mostly haemorrhagic) stroke.

\section{Diagnostic criteria}

A. Any new headache fulfilling criterion $\mathrm{C}$

B. Carotid endarterectomy has been performed

C. Evidence of causation demonstrated by at least 2 of the following:

1. Headache develops within 1 week of carotid endarterectomy

2. Headache resolves within 1 month after carotid endarterectomy

3. Headache is unilateral, on the side of the carotid endarterectomy, and has one of the following 3 characteristics:

a. Diffuse mild pain

b. Cluster headache-like pain occurring once or twice a day in attacks lasting $2-3 \mathrm{~h}$

c. Pulsating severe pain

D. Not better accounted for by another ICHD-3 diagnosis, and arterial dissection has been excluded by appropriate investigations

The study by Messert and Black [7] described 24 out of 57 patients developing headache. Nine patients had ipsilateral pain, of which 5 were described as cluster-like. One of them may have met the ICHD-criteria, but again not enough data was given.

In a case report by Björne et al. [8], a patient with a history of chronic cluster headache underwent a CEA after he had been free of attacks for 20 years. The day after surgery they recurred. This notes the importance of carotid hemodynamic changes along with the dysfunction of the sympathethic nervous system in the initiation of cluster headache.

\section{Discussion}

Although it would be possible that our 2 patients had idiopathic cluster headache, it is more likely that a relation exists between the CEA and the origin of cluster headache. Up to the present, after 2 years follow-up, neither of our patients had a second cluster period.

Headache following a CEA is frequently described and is mentioned separately in the ICHD-criteria [9] (Table 2), where 3 types are mentioned. The most frequently (up to 60\%) appearing headache is a mild diffuse isolated headache that occurs during the first few days after surgery; it is usually benign and self-limiting. The second type is a unilateral "cluster-like" pain (38\%) with attacks lasting 2-3 h, occurring once or twice a day, which resolve in about 2 weeks. The third type is part of the hyperperfusion syndrome, a unilateral pulsating and severe pain that occurs after an interval of 3 days after surgery. Patients actually developing cluster headache, which continued for several months, are not mentioned in these criteria, nor in our literature review, although some of the series we found suggested that some of the patients might have fulfilled the ICHD-criteria for cluster headache.

Cluster-like headache was described to occur frequently in 2 studies [6,7], but in more recent studies, it was not observed at all $[4,5]$. Therefore, the incidence of this headache seems lower than described in the ICHDIIIB criteria [9], unless we assume considerable underreporting. These studies show a more frequent form of ipsilateral headache. This is a mild headache, which does not resemble a cluster headache. The difference in these findings is most likely a problem of definition. There is no clear definition of cluster-like headache. Furthermore, studies had different follow-up durations and very few methodological data and patients characteristics were available.

Of interest in the present study is that cluster headache has been described in association with ICA dissection. In rare cases, carotid dissection presented with symptoms mimicking a cluster headache attack. In a recent review on secondary cluster headache, 11 out of 63 patients had a carotid/vertebral dissection [3]. A review in 2007 identified 6 patients with ICA dissection presenting with cluster-like features [10] and several more cases have been described [11-15]. In all these patients, headache attacks disappeared within 2 months.

The pathophysiology of primary cluster headache is still unclear. As described in the introduction, the TGA-reflex seems to play an important role. Direct injury to the carotid artery may cause the activation of this reflex [16]. Trigeminal nerve roots on the ICA, activated by surgical injury, will cause pain and reflex activation of parasympathetic nerves in the facial nerve. This will lead 
to the known autonomic symptoms and vasodilatation. Moreover, direct injury to the sympathetic plexus may, next to Horner's syndrome, cause vasodilatation, and thereby activate the TGA-reflex. Possibly post-operative vasodilatation caused by failed autoregulation may directly activate the TGA-reflex. These mechanisms may explain how a typical headache attack develops. However, cluster headache rarely develops following CEA. This also does not explain why damage to the carotid artery may cause episodic attacks. Studies in primary cluster headache show that the posterior hypothalamic region may play an important role in the pathophysiology of cluster headache [2]. Functional MRI, PET, and endocrine studies show altered activity in the posterior hypothalamus during attacks [17]. Furthermore, hypothalamic deep brain stimulation suppresses attacks in over $60 \%$ of therapy-resistant chronic cluster headache patients [2]. This hypothalamic dysfunction may lead to the disinhibition of the hypothalamo-trigeminal pathway and give rise to a permissive state, which seems necessary for a pain attack to begin.

With respect to our present study, an important question by Goadsby considering the pathophysiology of cluster headache is: "Is any peripheral drive necessary for cluster headache or is it entirely a brain disorder?" [16]. We suggest that damage to the carotid artery may lead to cluster headache attacks, including the typical frequency. Possibly in patients predisposed to cluster headache (e.g., hypothalamic dysfunction), injury to the ICA may disturb a balance that triggers attacks. As this damage resolves, as was seen in the carotid artery dissection case reports, attacks disappear. In patients with secondary cluster headache caused by a pituitary tumour, the headaches disappeared in all patients after treatment [3]. In the light of the above, an association with the compression of the ICA, which is located near the pituitary gland, could be considered. This supports the hypothesis that both a peripheral and central drive are necessary for cluster headache to develop. Current research, however, focuses on cluster headache as a brain disorder, rather than considering additional peripheral injury. This issue is extensively discussed in the article by Leone and Bussone [2]. The fact that patients, following trigeminal nerve root resection, can still develop cluster headache attacks is one of the findings that supports the "pure central" theory. Of course, the actual mechanism of a cluster headache attack is not clear and further research is still needed.

\section{Conclusion}

We described 2 cases of cluster headache following CEA and did not find any definite previous case descriptions in our literature review. Headache following CEA is a common symptom and this is usually a mild diffuse or ipsilateral headache, among which cluster-like headache is described. The relationship between the ICA and cluster headache is multiple in nature. Injury to the ICA may trigger cluster headache attacks, as shown by our 2 cases and the literature on ICA dissection. We argue that hypothalamic dysfunction and ICA damage are both required for cluster headache to develop.

\section{Statement of Ethics}

Written informed consent was obtained from the patients for publication of this case report and accompanying images.

\section{Disclosure Statement}

The authors declare that there is no conflict of interest.

\section{References}

1 Fischera M, Marziniak M, Gralow I, et al: The incidence and prevalence of cluster headache: a meta-analysis of population-based studies. Cephalalgia 2008;28:614-618.

2 Leone M, Bussone G: Pathophysiology of trigeminal autonomic cephalalgias. Lancet Neurol 2009;8:755-764.

3 Edvardsson B: Symptomatic cluster headache: a review of 63 cases. Springerplus 2014;3:64.

4 Albuquerque LA, Dellaretti M, Carvalho GT, et al: A prospective study of headache after carotid endarterectomy: incidence and clinical characteristics. Neurosci Med 2012;3:14-17.
5 Tehindrazanarivelo AD, Lutz G, PetitJean C, et al: Headache following carotid endarterectomy: a prospective study. Cephalalgia 1992; 12:380-382.

6 De Marinis M, Zaccaria A, Faraglia V, et al: Post-endarterectomy headache and the role of the oculosympathetic system. J Neurol Neurosurg Psychiatry 1991;54:314317.

7 Messert B, Black JA: Cluster headache, hemicrania, and other head pains: morbidity of carotid endarterectomy. Stroke 1978;9:559562.
8 Björne A, Hindfelt B, Havelius U: Recurrence of cluster headache after carotid thrombendarterectomy. Headache 1994;34: 230-233.

9 Headache Classification Committee of the International Headache Society (IHS): The international classification of headache disorders, 3rd edition (beta version). Cephalalgia 2013;33:629-808.

10 Rigamonti $\mathrm{A}$, Iurlaro $\mathrm{S}$, Reganati $\mathrm{P}$, et al: Cluster headache and internal carotid artery dissection: two cases and review of the literature. Headache 2008;48:467-470. 
11 Godeiro-Junior C, Kuster GW, Felicio AC, et al: Internal carotid artery dissection presenting as cluster headache. Arq Neuropsiquiatr 2008;66:763-764.

12 Tsivgoulis G, Mantatzis M, Vadikolias K, et al: Internal carotid artery dissection presenting as new-onset cluster headache. Neurol Sci 2013;34:1251-1252.
13 Hardmeier M, Gobbi C, Buitrago C, et al: Dissection of the internal carotid artery mimicking episodic cluster headache. J Neurol 2007; 254:253-254.

14 Tobin J and Flitman S: Cluster-like headaches associated with internal carotid artery dissection responsive to verapamil. Headache 2008; 48:461-466.

15 Candeloro E, Canavero I, Maurelli M, et al: Carotid dissection mimicking a new attack of cluster headache. J Headache Pain 2013;14:84.
16 Goadsby PJ: Pathophysiology of cluster headache: a trigeminal autonomic cephalgia. Lancet Neurol 2002;1:251-257.

17 Morelli N, Cafforio G, Maluccio MR, et al: Functional magnetic resonance imaging in episodic cluster headache. J Headache Pain 2009;10:11-14. 